\title{
The Quality Assessment of Performance in Intensive Care Units According to APACHE II Score
}

\author{
Manaf Abdi ${ }^{1}$, Shahram Tofighi ${ }^{2, *}$, Sharareh Niakan-Kalhori ${ }^{3}$, Abbas Ebadi ${ }^{4}$, Roohollah Zaboli ${ }^{1}$, Mohammad Hossein \\ Basirat $^{5}$, Esmaeil Feyzi ${ }^{1}$ \\ ${ }^{1}$ Department of Health Services Management, School of Health, Baqiyatallah University of Medical Sciences, Tehran, Iran \\ ${ }^{2}$ Health Management Research Center, Baqiyatallah University of Medical Sciences, Tehran, Iran \\ ${ }^{3}$ Department of Health Information Management, School of Allied Medical Sciences, Tehran University of Medical Sciences, Tehran, Iran \\ ${ }^{4}$ Behavioral Sciences Research Center, Baqiyatallah University of Medical Sciences, Tehran, Iran \\ ${ }^{5}$ Baqiyatallah Hospital, Tehran, Iran
}

*Corresponding Author: Shahram Tofighi, Assistant Professor, Health Management Research Center, Baqiyatallah University of Medical Sciences, Tehran, Iran. Email: shr_tofighi@yahoo.com

\begin{abstract}
Introduction: Improving the health level of patients is one of the most important purposes in intensive care units. In order to promote these units we need to measure their quality. To do so, some standards are needed in this area. The aim of this article was to study the mortality rate of the patients admitted to intensive care units with different APACHE scores.

Methods: This descriptive and retrospective study was conducted in Tehran, Iran. The sampling was census and all the patients who had been admitted to the intensive care unit in a hospital in Tehran in 2013 were studied in this study. The overall admitted patients were 350 that only 318 patients had completed the records and were involved in the study. Data was analyzed by using SPSS 15.

Results: Mortality and viability in these two groups were 122 and 196, respectively. Seven out of 68 with scores <15 and 9 out of 49 patients with scores between 19-16 died. Thirty three out of 103 patients who had scores between 30-20 died too and only 70 patients survived. In comparison with the standard scoring system, the mortality rate in our study was <19 which was lower than standard, but with scores $>20$, the mortality rate was $25 \%$ which was higher than standard values.

Conclusion: According to the obtained results, we can conclude that the quality of care in intensive care units is desirable. It can be said that according to the APACHE system the quality of healthcare is desirable in the Intensive Care Units. As a conclusion, in order to promote quality, more serious care is needed in patients with greater scores.
\end{abstract}

Keywords: Patients, Quality of Health Care, Intensive Care Unit, Mortality, APACHE

Article History: Received: 4 Feb. 2015; Accepted: 4 May. 2015; Online Published: 24 Aug. 2015

Cite this article as: Abdi M, Tofighi S, Niakan-Kalhori S, Ebadi A, Zaboli R, Basirat MH, et al. The quality assessment of performance in intensive care units according to APACHE II score. Int J Travel Med Glob Health. 2015;3(3):123-6.

\section{Introduction}

The intensive care unit is a specific location where the medical staff and equipment are used for the treatment and management of critically ill patients. A reasonable goal in this unit is to save the lives of patients with reversible situations. Since many factors play an important role in patient recovery, therefore the right selection of patients admitted to these units can have beneficial effects on treatment processes [1]. Nowadays, therapists can treat many diseases by using new and advanced technologies and methods which leads to the longer survival of patients.

The need to assess quality of services provided in intensive care units and comparing these services with standard ones and using data to improve the care outcomes of patient led to a criterion as Acute Physiology, Age and Chronic Health Evaluation Acute that its acronym is APACHE (Acute Physiology and Chronic Health Evaluation). Humans have always been interested in the quality of services and production. The importance of quality in industries was considered in the 1940-1950s and was proposed in nursing sciences in the 1980s. The Joint Commission Accreditation Healthcare Organization believes that quality is: "An acceptable level of health services provided to individuals and populations that increase the possibility of desired health outcomes and are consistent with the professional knowledge of the day". Every year, more than 2 million deaths occur in Intensive Care Units of USA hospitals which can be reduced by more than $30 \%$ [2].
Other than monitoring and treating critically ill patients, intensive care units' doctors are also responsible for both predicting the patients' outcomes and identifying and distinguishing them. This is because hospitalizing is not necessarily useful for all patients. Actually in some patients it will just lead to a more comfortable death [1]. Using the severity-of-disease, the classification system can be a guidance for physicians to evaluate the patients' outcome and to estimate the chance of recovery. The determination of prognosis systems can also help estimate the psychological instability in patients' admitted in hospitals. The severity of illness scoring and predicting the mortality rate can also be done in besides to the evaluation. Thereby the chance of the survival of patients will be assessed more accurately by physicians and leads to longer survival of patients [3].

APACHE II is a severity-of-disease classification system (Knaus et al, 1985) that includes 12 physiological measurements. This system is still used to predict the admitted patients' treatment process in hospitals especially in intensive care units due to its easy collecting of information through routine checkups and reducibility [4]. In fact, because of the differences among patients in the same ward, having a specific estimation system for every patient in every unit is necessary. In addition to prioritizing patients to receive appropriate care facilities, the physicians can have a more accurate judgment to predict mortality rates, occupying the hospital beds and patients' treatment process. This system 
can also be a criterion for measuring the standard rate of intensive care units and can be compared with global standards [1].

According to the standards table, if the APACHE II score is $0-15,16-19,20-30$ and above 30 , the possibility of mortality will be $15,10,35$ and 75 , respectively [5].

This study aimed to investigate the diagnosis possibility in patients admitted to intensive care units in Tehran and was conducted by using APACHE II. During different studies, assessment and scoring by APACHE has been conducted in some of the medical and educational centers in Iran. In this study we decided to estimate the mortality rate in patients admitted to intensive care units by using the severity of illness scoring systems.

\section{Methods}

This study is a descriptive and retrospectively study. The records of all the patients which had been admitted to this hospital's intensive care unit in 2013 were used. Patients who were under 18, those who had CPR (Cardiopulmonary Resuscitation), those who were admitted to the intensive care unit for less than 24 hours and also brain dead patients were excluded from this study. Data was collected by using the checklists in accordance with the APACHE II. APACHE II checklist has 2 sections: the first section includes the personal identification and general and demographic questions such as age, gender, consciousness level, need for mechanical ventilation and length of stay in the intensive care unit, and the second section includes clinical and paraclinical data. Additionally, as the emphasis of this study was on ward management, our focus was on the performance during hospitalization.

Clinical and para-clinical parts are also divided into three sections. 11 items and the Glasgow Coma Scale are used to measure physiological variables. Minimum and maximum scores of each question in the physiological dimension were 0 and 4, respectively. These 11 items included: Temperature, Mean arterial pressure, Heart rate, Respiratory rate per 1 minute, Sodium and Potassium amount, Creatinine $\mathrm{pH}, \mathrm{PaO} 2$ serum, Hematocrit, White blood cell count and $\mathrm{pH}$ arterial. The minimum and maximum scores in the physiological dimension were 0 and 59, respectively [6].

The score of the second section was obtained through patients' age group. For the patients older than 44 years old, the minimum and maximum scores were 0 and 6 , respectively. The third part was related to the evaluation of chronic disease and chronic renal failure in one or multiple organs of patients which included heart, lungs, kidneys, liver and immune system. The APACHE score was obtained from the total scores of these three parts (minimum score is 0 and maximum is 71). The mortality score predicts on the basis of percent. It is important to collect the data in the first $24 \mathrm{~h}$ of hospitalization which was considered in this study. Mortality was considered as deaths which occurred during the patient's stay in hospital. The observed mortality rate was compared with the predicted mortality rate.

To avoid biased interpretations:

1. Apache point was calculated in the first $24 \mathrm{hrs}$ of hospitalization.

2. Patients who had been hospitalized more than $24 \mathrm{hrs}$ and received controlling and therapeutic intervention were excluded from this study.

3. Patients who had been hospitalized due to brain death, were excluded from this study.

The results were analyzed in SPSS version 15 and t-test and chi-square test were calculated. According to APACHE scores, the percent of mortality in each group was also calculated. The reason for choosing this test was the fact that this study was done on two independent groups (improved and died patients) and that the normality of samples were ascertained by Kolmogrov Smirnov test.

\section{Results}

Among the 350 patients, 318 cases were included in this study. Among them, $50.6 \%$ were male and $49.4 \%$ were female. It was also recorded that $50.3 \%$ of the patients were admitted for 5 days and $49.7 \%$ of them had stayed in the hospital for more than 5 days. The distribution of age was: $4 \%$ from hospitalized patients in $10-19$ year olds, $13 \%$ in 20 34 year olds, $15 \%$ in $35-54$ year olds and $68 \%$ in those over 55 years old. It can also be mentioned that $38 \%$ of the studied patients died and $62 \%$ of them finally survived.

Table 1. Results of patients on the basis of APACHE II

\begin{tabular}{|c|c|c|c|c|}
\hline & APACHE II Score & Percentage of Mortality & Percentage of Standard Death & Hospital Condition \\
\hline 1 & $0-15$ & 9 & 10 & Up to standard \\
\hline 2 & $16-19$ & 14 & 15 & Up to standard \\
\hline 3 & $20-30$ & 43 & 35 & Lower than standard \\
\hline 4 & $31 \leq$ & 85 & 75 & Up to standard \\
\hline
\end{tabular}

Table 2. Comparison of mortality based on APACHE II

\begin{tabular}{|c|c|c|c|c|}
\hline APACHE II Score & $\mathbf{N}(\%)$ & $\mathbf{N}(\%)$ & Total & P Value \\
\hline $0-15$ & $61(32.8)$ & $7(5.3)$ & $68(21.4)$ & 0.57 \\
\hline $16-19$ & $40(21.5)$ & $9(6.8)$ & $49(15.4)$ & 0.77 \\
\hline $20-30$ & $70(37.7)$ & $33(25)$ & $103(32.3)$ & 0.001 \\
\hline $31 \leq$ & $15(8)$ & $83(62.8)$ & $98(30.9)$ & 0.001 \\
\hline Total & $186(58.5)$ & $132(41.5)$ & $318(100)$ & 0.001 \\
\hline
\end{tabular}

As shown in the table above, mortality rates for 0-15 and 16-19 scores are higher than standards and for the scores 2030 and $31<$ are less than standard rates. In order to achieve a satisfactory level of quality, the hospital needs to provide more suitable healthcare services to end up with higher Apache points.

The frequency of distribution related to the duration of hospitalization between the dead and survival groups showed that among the survival group, $64 \%$ were hospitalized for up to 5 days and $36 \%$ were hospitalized more than 5 days. Among the dead group, $76 \%$ were hospitalized for up to 6 days and $24 \%$ were hospitalized more than 5 days. No significant differences were found in the duration of hospitalization in between the two groups $(\mathrm{P}=0.345)$. The number of dead and survival men were more than women, but the sex differences were not significant $(\mathrm{P}=0.526)$. There was no significant relationship between the diagnosis at admission and mortality rate $(\mathrm{P}=0.787)$. 
The relation between the duration of hospitalization, life status, type of trauma, APACHE score and gender was statically significant $(\mathrm{p}=0.001, \mathrm{p}=0.023, \mathrm{p}=0.00, \mathrm{p}=0.044)$. The mortality rate in patients with an APACHE score of 0 15 was $9 \%$ while this score is $13 \%$ in standard rates. This amount for 16-19 was 14 percent which was lower than standard. For 20-30 and above 30 was 43 and 85 percent which was higher than standard rates (the standard rate is $35 \%$ and $75 \%$, respectively).

\section{Discussion}

Predicting the mortality rate in trauma patients with the APACHE scoring system has been a successful performance for many years [7]. The mortality rate in this study was $22.7 \%$, while this amount was $27.9 \%$ in Schein et al.'s study. Also, Kulkarni reported 11 and 16 percent, respectively [8, 9]. In a study conducted by Dossett et al. on patients that had been admitted to intensive care units, it was mentioned that the mortality rate was 14 percent [10]. Due to low hospital standards, this amount is justifiable. On the other hand, in this study patients were studied that had higher risk of death. In comparison to the standard table, our table showed that the mortality rate in patients with the scores < 15 was $9 \%$ which is close to the standard score $(10 \%)$. Mohammadi et al. reported that the mortality rate in this group is zero [11]. Also, in a study which was conducted by Rahimzade et al. on the Rasool Akram Hospital showed that this amount is 8 percent [2].

The mortality rate in the scores between 16 and 19 was 14 percent that was lower than standard rates $(15 \%)$. The mortality rate in the scores of 30 and above 30 were also 43 and $85 \%$ respectively which were higher than standard (35 and 75 percent).

This study showed that the mortality rate for the scores $15<$ are lower than standard rates. The studies that have been conducted in Iran show the opposite results in some cases. By increasing the APACHE score, the mortality rate also increases. This study showed that the APACHE score in both the survived and dead groups have no significant differences. However, the mortality rate in the different APACHE scores should be revised in our country and predictions should be based on the results of researches conducted in this area. Foreign studies showed that the APACHE scores is useful in predicting the mortality rate, but this prediction is not the same for all patients [12, 13]. Increasing the differences among the dead group in our study and other studies may be due to the hospital standards such as medical equipment in intensive care units, adjustment of manpower, shift work and differences in policies in different hospitals [13].

This study showed that this system is useful for patients whom were admitted to intensive care units. In other studies that compared the APACHE II with APACHE III, TISS and SAPS, found that the APACHE II has a much higher predictive power and is good for analyzing the quality in intensive care units [14]. The studies showed that the APACHE II alone is not useful for predicting the mortality rate, the severity of disease classification and length of stay, but it will be helpful in managing and providing the treatment methods, comparative effectiveness of treatments, making decisions for changing treatment and comparing the performances and quality of services provided. Nevertheless, the use of APACHE II in other sections is not yet known [15, 16]. The usefulness of this scoring system in classifying the patients admitted to intensive care units or better managing the patients that have had surgery has been confirmed by some studies [17].

Many factors affect the accuracy of prediction of mortality rates. These factors include limitations of APACHE II that are due to a lot of components both in measuring and the individual differences (ethnicity, cultural, economic and social) of patients studied in this article in compared to patients evaluated for validation of the tool [18]. The other factors may be related to criteria that are for admitting patients in intensive care units. The number of beds in hospitals will also effect results [6]. According to the limited beds in intensive care units in our country, using this scoring can be helpful for patients whom have higher scores. This is because patients are prioritized and those who are in higher risk can have better outcomes by getting admitted to intensive care units.

There are many indicators to classify the severity of diseases. This is while in many hospitals and training centers, patients admitted to intensive care units are still evaluated by using traditional methods such as GCS [6]. As shown in this study, there is significant differences between GCS scores in survival groups and dead groups. This result indicates that GCS can be used for the admission of patients because this criterion can be measured simply and can be checked in early hours of hospitalization. Dossett et al. also showed the same relation [10]. This study indicated that there is no significant relation between age, gender, length of stay and the diagnosis at admission and mortality of patients admitted to intensive care units. This result has been confirmed in many other studies $[19,20]$.

\section{Conclusion}

The results showed that the mortality rate in intensive care units in patients with the scores $16<$ is lower than the standard rate. These results can be a guidance for predicting the mortality rate in this unit in terms of the APACHE score and can be effective in the decision making processes of practitioners. This big difference with standard scores can be due to the differences between treatments and cares in Iran's medical centers and other countries. The main reasons can be: lack of skilled manpower in emergency cases, lack of experience and not enough care in managing and nursing critically ill patients and lack of appropriate equipment and physical space. According to the results, care and treatment interventions in the hospital should be revised by using a prospective study and a larger sample size. Also, the level of quality should reach standard levels in order to reduce differences.

According to the limited number of beds in Iranian hospitals, regular use of this scoring in intensive care units will be helpful in determining the priority of patients whom have more need for care (have a higher APACHE score). Patients who obtain lower scores and have a lower risk can be placed in the next priority in the allocation of intensive care unit beds. Thus, patients who needed intensive care and had a higher scoring could indicate better outcomes after treatment in intensive care units.

\section{Suggestion}

1. Structural standards such as human force, physical environment, equipment and so on must be observed by all work shifts.

2. A model of quality improvement standards and ranking must be implemented in the ward.

3. Safety indices must he determined and monitored in the 
ward.

4. Triage and assessment of the patients must be performed based on the APACH2 method when the patient is admitted.

5. The nurses and health service staff must receive periodical and annual trainings.

6. Scoring and assessing the patients under $\mathrm{APACH} 2$ standards must be computerized by developing software; this minimizes human errors.

\section{Limitations}

1. Incomplete file: to avoid biased interpretation, the incomplete files were excluded.

2. Lack of pre-clinical information registration system: to deal with this, the needed information was collected from the patients' file.

3. Some patients were hospitalized more than 24hrs: after $24 \mathrm{hrs}$ hospitalizations, the patients have received health care services and their condition is stabilized; therefore they were excluded from the study.

\section{Acknowledgments}

The authors would like to thank the responsible minister of medical records for offering extremely useful information related to this study.

\section{Authors' Contributions}

All authors were involved in the study design and result interpretation. All authors confirmed the final draft before submission.

\section{Financial Disclosure}

The authors declared no financial disclosure.

\section{Funding/Support}

Not declared

\section{References}

1. Rothen HU, Stricker KH, Heyland DK. Family satisfaction with critical care: measurements and messages. Curr Opin Crit Care. 2010;16(6):623-31.

2. Rahimzadeh P, Taghipur Anvari Z, Hassani V. Estimation of mortality rate of patients in surgical intensive care unit of HazratRasul hospital. Hakim Res J. 2008;11(1):22-8. Persian

3. Khwannimit B, Geater A. A comparison of APACHE II and SAPS II scoring systems in predicting hospital mortality in Thai adult intensive care units. J Med Assoc Thai. 2007;90(4):643-52.
4. Matic I, Titlic M, Dikanovic M, Jurjevic M, Jukic I, Tonkic A. Effects of APACHE II score on mechanical ventilation; prediction and outcome. Acta Anaesthesiol Belg. 2007;58(3):177-83.

5. Le Gall JR. The use of severity scores in the intensive care unit. Intensive Care Med. 2005;31(12):1618-23.

6. Winn HR, Youmans JR. Youmans neurological surgery: Saunders; 2004.

7. Soleimani MA, Masoudi R, Bahrami N, Qorbani M, Sadeghi T. Predicting mortality rate of patients in critical care unit using APACHE-II index. J Gorgan University Med Sci. 2010;11(4):64-9.

8. Schein M, Gecelter G, Freinkel Z, Gerding H. APACHE II in emergency operations for perforated ulcers. Am J Surg. 1990;159(3):309-13.

9. Kulkarni SV, Naik AS, Subramanian N, Jr. APACHE-II scoring system in perforative peritonitis. Am J Surg. 2007;194(4):549-52.

10. Dossett LA, Redhage LA, Sawyer RG, May AK. Revisiting the validity of APACHE II in the trauma ICU: improved risk stratification in critically injured adults. Injury. 2009;40(9):993-8.

11. Mohammadi H, Haghighi M. The Assessment of mortality in ICU with different APACHE II scores. J Guilan Univ Med Sci. 2006;15(59):85-90. Persian

12. Knaus WA, Wagner DP, Draper EA, Zimmerman JE, Bergner M, Bastos PG, et al. The APACHE III prognostic system. Risk prediction of hospital mortality for critically ill hospitalized adults. Chest. 1991;100(6):1619-36

13. Van Le L, Fakhry S, Walton LA, Moore DH, Fowler WC, Rutledge R. Use of the APACHE II scoring system to determine mortality of gynecologic oncology patients in the intensive care unit. Obstet Gynecol. 1995;85(1):53-6.

14. Gupta R, Arora VK. Performance evaluation of APACHE II score for an Indian patient with respiratory problems. Indian J Med Res. 2004;119(6):273-82.

15. Ho YP, Chen YC, Yang C, Lien JM, Chu YY, Fang JT, et al. Outcome prediction for critically ill cirrhotic patients: a comparison of APACHE II and Child-Pugh scoring systems. J Intensive Care Med. 2004;19(2):105-10.

16. Moreno RP, Metnitz PG, Almeida E, Jordan B, Bauer P, Campos RA, et al. SAPS 3--From evaluation of the patient to evaluation of the intensive care unit. Part 2: Development of a prognostic model for hospital mortality at ICU admission. Intensive Care Med. 2005;31(10):1345-55.

17. Neishaburi M, Raies Dana N, Ghorbani R, Sadeghi T. Examining the nursing care quality from the viewpoints of nurses and patients of therapeutic-educational centers of the city of Semnan. Koomesh. 2010;12(2):134-43.

18. Ratanarat R, Thanakittiwirun M, Vilaichone W, Thongyoo S, Permpikul C. Prediction of mortality by using the standard scoring systems in a medical intensive care unit in Thailand. J Med Assoc Thai. 2005;88(7):949-55.

19. Atiyat B, Kloub A, Abu-Ali H, Massad I. Clinical Sense in the Prediction of Surgical/Trauma Intensive Care Mortality. European J Sci Res. 2009;30(2):265-71.

20. Bo M, Cacello E, Ghiggia F, Corsinovi L, Bosco F. Predictive factors of clinical outcome in older surgical patients. Arch Gerontol Geriatr. 2007;44(3):215-24. 